\title{
Cutaneous leishmaniasis mimicking sarcoidosis in Libyan patient: A case report
}

\begin{abstract}
Leishmaniasis is vector-borne disease caused by parasitic protozoans belonging to the genus Leishmania and is transmitted by infected phlebotomine sand flies. Sarcoidosis is a multisystemic disorder of unknown cause characterized by the formation of immune granulomas in affected organs. Clinical symptoms, severity and evolution of sarcoidosis are highly heterogeneous and can lead to other diseases with similar clinical and pathologic presentations. In this report we present a case of 77 years-old diabetic Libyan male, with chronic erythematous indurated plaques, and nodules on the face. The patients were treated by multiple physicians with topical and systemic corticosteroids for 25 years without improvement. Microscopic examination of Giemsa stained smears from all lesions showed numerous Leishmania amastigotes in and outside monocytes. Leishmania tropica was identified as causative species. The patient was treated with combination of oral rifampicin $(600 \mathrm{mg} /$ day) and isoniazide (300 mg/day) and followed up for 9 months until skin-slit smears and PCR turned negative. In conclusion, CL can be misdiagnosed clinically with any granulomatous skin lesions which are compatible with sarcoidal type granuloma. Molecular diagnosis of CL by implementing Leishmania-specific PCR approaches should be performed routinely in any granulomatous skin lesion.
\end{abstract}

Volume 8 Issue 5 - 2020

\author{
Ahmad Amro,' Hamida Al-Dwibe, ${ }^{2}$ Ali \\ Lashhab, ${ }^{3}$ Esseid Elzubi, ${ }^{2}$ Walid K Saadawi, ${ }^{4}$ \\ Aisha Gashout ${ }^{5}$ \\ 'Faculty of Pharmacy, AL-Quds University, Palestine \\ ${ }^{2}$ Dermatology Department, Tripoli Central Hospital, Libya \\ ${ }^{3}$ Dermatology Department, Ber-Austa Milad Hospital, Libya \\ ${ }^{4}$ Libyan National Centre for Infectious Diseases and Control, \\ Libya \\ ${ }^{5}$ Faculty of Medical Technology-Pathology Department, \\ University of Tripoli, Libya
}

\begin{abstract}
Correspondence: Dr.Ahmad Amro, Faculty of Pharmacy, Alquds University, Main Campus, P.O. Box 5100 , Abu Dis, Jerusalem, Palestine, Tel +970(2)27904 I2, Fax +970(2)27904 I3, Email aaro@pharm.alquds.edu, ahmdymm@hotmail.com
\end{abstract}

Received: September 30, 2020 | Published: October 12, 2020

\section{Introduction}

Leishmaniasis is a vector-borne disease caused by parasitic protozoans belonging to the genus Leishmania and is transmitted by infected phlebotomine sand flies. The clinical manifestations is polymorphic and range from cutaneous (CL) and muco-cutaneous leishmaniasis which is characterized by localized lesions on the skin and in mucous membranes, to visceral leishmaniasis which is the most severe form and mostly fatal in many developing countries if left untreated. ${ }^{1}$ These manifestations (dermotropic and viscerotropic) depend on the causative Leishmania species, ${ }^{2-4}$ and the immunological response of the infected host. ${ }^{5} \mathrm{CL}$ may appear as unusual clinical and histopathological forms and mimic other co-morbidities that causes diagnostic challenge and may lead to misdiagnosis.

Sarcoidosis is a multi-systemic disorder of unknown cause characterized by the formation of immune granulomas in affected organs. The most affected organs are the lungs and lymph nodes. However, it can affect every organ of the body from the skin and eyes to be most common extra pulmonary organs that develop serious clinical manifestations ${ }^{6-8}$ Estimated mortality attributable to sarcoidosis is between $0.5 \%$ and $5 \%$. Most of these deaths result from pulmonary, cardiac and central nervous system. ${ }^{9,10}$ Clinical symptoms, severity and evolution of sarcoidosis are highly heterogeneous and is affiliated to other diseases with similar clinical and pathologic presentations. The majority of cases are time limiting and may show spontaneous resolution within 1-3 years. However, serious and prolonged course may be experienced in some patients. ${ }^{9}$ Sarcoidal granuloma in this study, we reported for the first time in Libya a patient with CL symptoms mimicking sarcoidosis.

\section{Case report}

In this report we present a case of 77 years-old diabetic Libyan male, with chronic erythematous indurated plaques, and nodules on the face and ears for more than 25 years duration. According to his clinical and histological characteristics, the patient was misdiagnosed with sarcoidosis and was treated by multiple physicians with topical and systemic corticosteroids for 25 years without improvement. The patient was admitted to our department in March 2015. On examination there were several small painless popular lesions with indurations. The lesions showed central ulceration with erythematous borders over the forehead and cheek (Figure 1A). Skin smears and biopsies were taken under local anesthesia and sent for Lab analysis. Microscopic examination of Giemsa stained smears from all lesions showed numerous Leishmania amastigotes in and outside monocytes (Figure 2). Leishmania tropica was identified as causative species by amplification of the Internal transcribed spacer 1 (ITS1) PCR followed by RFLP with HaeIII restriction enzyme. The patient was treated with combination of oral rifampicin $(600 \mathrm{mg} /$ day $)$ and isoniazide $(300 \mathrm{mg} /$ day) and followed up for 9 months until skin-slit smears and PCR turned negative (Figure 1B). The cutaneous lesions of the face disappeared and there has been no evidence of recurrence after three years (Figure 1C). 

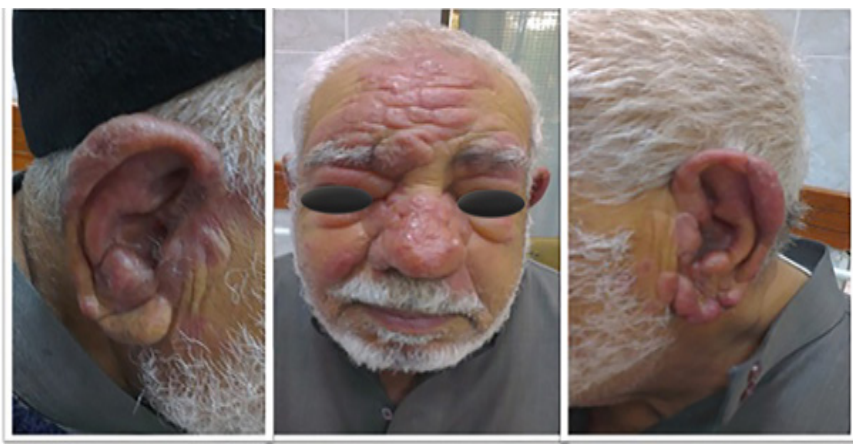

(A) March 2015
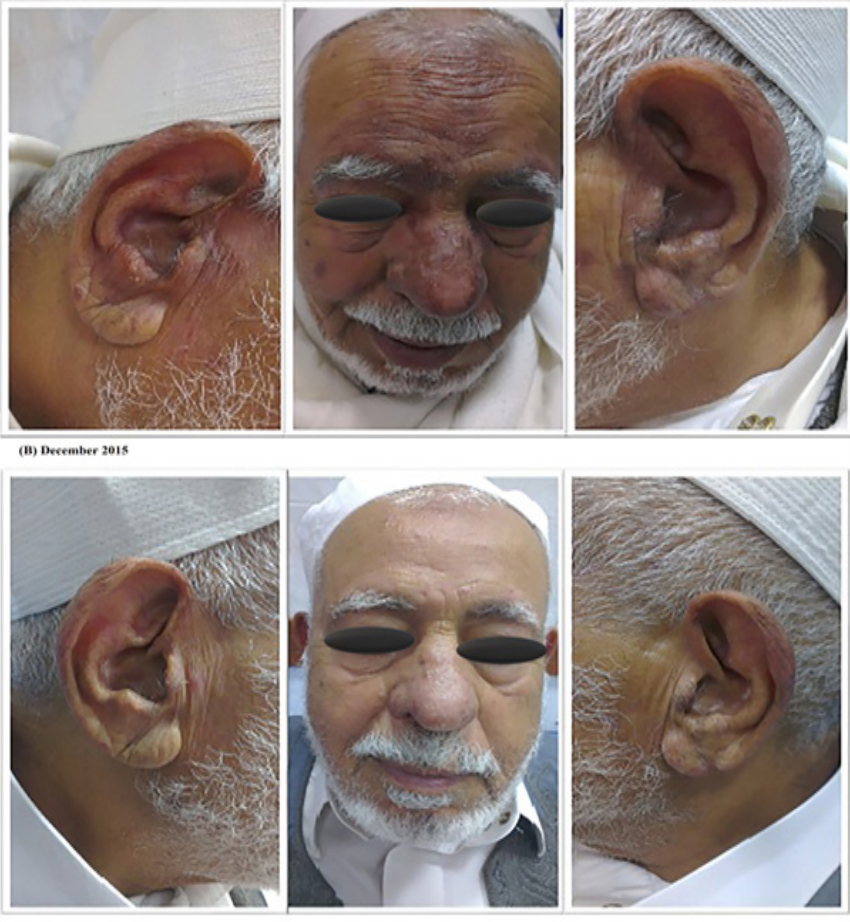

(C) Octaber 2018

Figure I (A) the patient was admitted on March 2015 with small painless lesions with central ulcerations and erythematous borders over the forehead and cheek. (B) after 9 months of treatment with oral rifampicin and isoniazide. (C) the patient was completely recovered after 3 years of follow up.

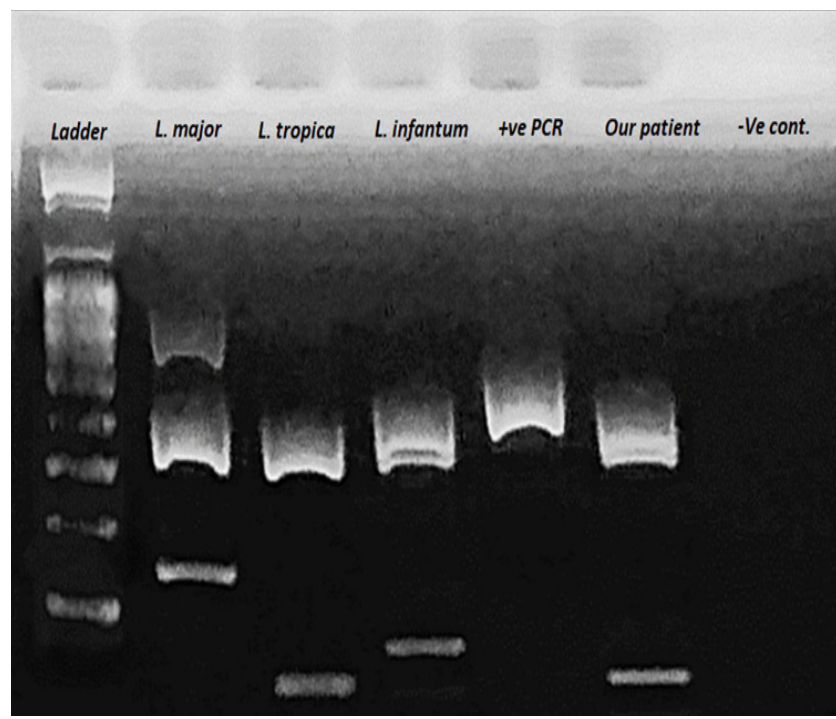

Figure 2 Restriction fragment length polymorphism (RFLP) profile of amplified ITSI region with the Hae III restriction enzyme. From the left: Lane I is I00 bp marker, lane 2, 3 and 4 are digested positive controls of L. major, L. tropica and L. infantum respectively. Lane 5 is an undigested PCR product of the ITSI region showed the characteristic band of about $300 \mathrm{bp}$, and lane 6 is the digested PCR product from our patient identified as L. tropica. 


\section{Discussion}

Sarcoidal granuloma may appear with heterogeneous presentations that mimic several infectious diseases such as tuberculoid leprosy, lupus vulgaris, lupoid rosacea, granuloma annulare and coetaneous leishmaniasis. ${ }^{10-12}$ This appearance is challenging in deferential diagnosis between theses infectious diseases and sarcoidosis which should be always remembered as diagnosis of exclusion. The emergence and development of modern diagnostic techniques has led to better discrimination of these cases. Patients who were previously diagnosed with sarcoidosis were tested positive fordifferent coinfections. ${ }^{13-16}$ However, few cases of CL associated with sarcoidosis have been described previously. ${ }^{17-19}$ Leishmania parasites were isolated from significant number of patients with single and/or multiple nodular lesions of the skin. Chronic forms of nodular skin lesions represent a diagnostic dilemma in clinical settings and from a histological aspect. ${ }^{17,20}$ In case of unusual CL forms and coinfections, Leishmania amastigotes are either difficult to trace in these chronic skin lesions or is altogether absent. In clinical practice, these forms may be misinterpreted as sarcoidosis, particularly in cases where microscopic screening for Leishmania amastigotes from skin-slit smears from these lesions reported negative. This misdiagnosis of $\mathrm{CL}$ with other mimicking diseases such as sarcoidosis has led to implementation of treatment strategy which is in complete contradiction to $\mathrm{CL}$ treatment. Our patient received a long-term therapy of corticosteroids which significantly modulated the immune response and increasing the patient's susceptibility to parasitic infections such as CL. ${ }^{21}$ Administration of immunosuppressive therapies such as corticosteroids has been described as a risk factor associated with CL reactivation. ${ }^{21}$ Previous experimental studies have shown that $\mathrm{CL}$ is usually being controlled predominately by T-cell mediated immunity with a preferential Th1 pattern. ${ }^{22}$ Hence, the incidence of CL in these immune-compromised patients may be due to inadequate cellular immune response. Moreover, the causative agent of CL in our patient belonged to L. tropica species. To the best of our knowledge, the phenomenon of CL mimicking sarcoidosis due to $L$. tropic has not been reported before in Libya.The advancement of molecular diagnostic techniques for CL in Libya has led to an accurate and rapid detection of Leishmania amastigotes where microscopic and histological diagnosis failed to identify them. Moreover, it has enabled a precise identification of the causative species, better deferential diagnosis from comorbidities, and consequently better treatment and recovery of patients. ${ }^{23-25}$ The sensitivity of molecular diagnostic methods for CL including kDNA and ITS1 PCR was higher compared to other diagnostic approaches. These differences in sensitivity become more significant in patients with chronic forms of nodular skin lesions. ${ }^{17,22}$

In conclusion, $\mathrm{CL}$ can be misdiagnosed clinically with any granulomatous skin lesions which are compatible with sarcoidal type granuloma. From Histopathological point of view, CL may be misinterpreted as sarcoidosis especially when Leishmania amastigotes are not seen microscopically. Considering their epidemiological features, endemicity, and geographical distribution, molecular diagnosis of CL by implementing Leishmania-specific PCR approaches should be performed routinely in any granulomatous skin lesion.

\section{Acknowledgments}

None.

\section{Conflicts of interest}

Authors declare that there is no conflict of interest.

\section{References}

1. Desjeux P. Worldwide increasing risk factors for leishmaniasis. Med Microbiol Immunol. 2001;190(1-2):77-79.

2. Chargui N, Amro A, Haouas N, et al. Population structure of Tunisian Leishmania infantum and evidence for the existence of hybrids and gene flow between genetically different populations. Int $J$ Parasitol. 2009;39(7):801-811.

3. Seridi N, Amro A, Kuhls K, et al. Genetic polymorphism of Algerian Leishmania infantum strains revealed by multilocus microsatellite analysis. Microbes Infect. 2008;10(12-13):1309-1315.

4. Amro A, Hamdi S, Lemrani M, et al. Moroccan Leishmania infantum: genetic diversity and population structure as revealed by multi-locus microsatellite typing. PLoS One. 2013;8:e77778.

5. Ready PD. Epidemiology of visceral leishmaniasis. Clin Epidemiol. 2014;6:147-154.

6. Siltzbach LE, James DG, Neville E, et al. Course and prognosis of sarcoidosis around the world. Am J Med. 1974;57(6):847-852.

7. Baughman RP, Lower EE, du Bois RM. Sarcoidosis. Lancet. 2003;361(9363):1111-1118.

8. Rizzato G, Palmieri G, Agrati AM, et al. The organ-specific extrapulmonary presentation of sarcoidosis: a frequent occurrence but a challenge to an early diagnosis. A 3-year-long prospective observational study. Sarcoidosis Vasc Diffuse Lung Dis. 2004;21(2):119-126.

9. Hunninghake GW, CostabelU, Ando M, et al.ATS/ERS/WASOG statement on sarcoidosis. American Thoracic Society/European Respiratory Society/ World Association of Sarcoidosis and other Granulomatous Disorders. Sarcoidosis Vasc Diffuse Lung Dis. 1999;16(2):149-173.

10. ZahafA, Turki H, Masmoudi A. Tubercular lupus. Ann Dermatol Venereol. 2004;131(5):503-507.

11. Landau M, Srebrnik A, Brenner S. Leishmaniasis recidivans mimicking lupus vulgaris. Int J Dermatol. 1996;35(8):572-573.

12. Weissler JC. Southwestern internal medicine conference: sarcoidosis: immunology and clinical management. $\mathrm{Am} \mathrm{J} \mathrm{Med} \mathrm{Sci.}$ 1994;307(3):233-245

13. Li N, Bajoghli A, Kubba A, et al. Identification of mycobacterial DNA in cutaneous lesions of sarcoidosis. J Cutan Pathol. 1999;26(6):271-278.

14. Almenoff PL, Johnson A, Lesser M, et al. Growth of acid fast L forms from the blood of patients with sarcoidosis. Thorax. 1996;51(5):530-533.

15. Ikonomopoulos JA, Gorgoulis VG, Zacharatos PV, et al. Multiplex polymerase chain reaction for the detection of mycobacterial DNA in cases of tuberculosis and sarcoidosis. Mod Pathol. 1999;12(9):854-862.

16. Nilsson K, Pahlson C, Lukinius A, et al. Presence of Rickettsia helvetica in granulomatous tissue from patients with sarcoidosis. J Infect Dis 2002;185(8):1128-1138.

17. Boer A, Blodorn-Schlicht N, Wiebels D, et al. Unusual histopathological features of cutaneous leishmaniasis identified by polymerase chain reaction specific for Leishmania on paraffin-embedded skin biopsies. $\mathrm{Br} J$ Dermatol. 2006;155(4):815-819.

18. Hill PA. A case of granulomatous dermatitis: cutaneous leishmaniasis. Pathology. 1997;29(4):434-436.

19. Wermert D, Lamblin C, Wallaert B, et al. Pulmonary sarcoidosis with cutaneous leishmaniasis. Sarcoidosis Vasc Diffuse Lung Dis. $1999 ; 16: 228-229$ 
20. Convit J, Ulrich M, Perez M, et al. A typical cutaneous leishmaniasis in Central America: possible interaction between infectious and environmental elements. Trans $R$ Soc Trop Med Hyg. 2005;99(1):13-17.

21. Mueller MC, Fleischmann E, Grunke M, et al. Relapsing cutaneous leishmaniasis in a patient with ankylosing spondylitis treated with infliximab. Am J Trop Med Hyg. 2009;81(1):52-54.

22. Farrell JP, Muller I, Louis JA. A role for Lyt-2+ T cells in resistance to cutaneous leishmaniasis in immunized mice. $J$ Immunol. 1989;142(6):2052-2056.
23. Al-Dwibe H, Gashout A, Morogum AM, et al. Contact dermatitislike cutaneous leishmaniasis in a Libyan HIV patient. Parasit Vectors. 2014;7:401.

24. Amro A, Gashout A, Al-Dwibe H, et al. First molecular epidemiological study of cutaneous leishmaniasis in Libya. PLoS Negl Trop Dis. 2012;6(6):e1700.

25. Amro A, Al-Dwibe H, Gashout A, et al. Spatiotemporal and molecular epidemiology of cutaneous leishmaniasis in Libya. PLoS Negl Trop Dis. 2017;11(9):e0005873. 